\title{
THE RESURGENCE PROPERTIES OF THE LARGE ORDER ASYMPTOTICS OF THE ANGER-WEBER FUNCTION II
}

\author{
GERGő NEMES
}

Abstract. In this paper, we derive a new representation for the Anger-Weber function, employing the reformulation of the method of steepest descents by C. J. Howls (Howls, Proc. R. Soc. Lond. A 439 (1992) 373-396). As a consequence of this representation, we deduce a number of properties of the large order asymptotic expansion of the Anger-Weber function, including explicit and realistic error bounds, asymptotic approximations for the late coefficients, exponentially improved asymptotic expansions, and the smooth transition of the Stokes discontinuities.

Mathematics subject classification (2010): 41A60, 30E15, 34M40.

Keywords and phrases: asymptotic expansions, Anger-Weber function, error bounds, Stokes phenomenon, late coefficients.

\section{REFERENCES}

[1] M. V. BERRY, Uniform asymptotic smoothing of Stokes' discontinuities, Proc. Roy. Soc. London Ser. A 422 (1989), 7-21.

[2] A. R. Booker, A. Strömbergsson And H. Then, Bounds and algorithms for the K-Bessel function of imaginary order, LMS J. Comput. Math. 16 (2013), 78-108.

[3] R. B. Dingle, Asymptotic Expansions: Their Derivation and Interpretation, Academic Press, London/New York, 1973.

[4] C. J. Howls, Hyperasymptotics for integrals with finite endpoints, Proc. Roy. Soc. London Ser. A 439 (1992), 373-396.

[5] C. S. MEIJER, Über die asymptotische Entwicklung von $\int_{0}^{\infty-i(\arg w-\mu)} e^{v z-w \sinh z} d z \quad\left(-\frac{\pi}{2}<\mu<\frac{\pi}{2}\right)$ für grosse Werte von $|w|$ und $|v|$, Proc. Kon. Akad. Wet. Amsterdam 35 (1932), pp. 1170-1180 and 1291-1303.

[6] G. NEMES, The resurgence properties of the large order asymptotics of the Hankel and Bessel functions, submitted, Anal. Appl. 12 (2014), 403-462.

[7] G. NEMES, The resurgence properties of the large-order asymptotics of the Anger-Weber function I, J. Classical Anal. 4, 1 (2014) 1-39.

[8] F. W. J. OLVER, Error bounds for stationary phase approximations, SIAM J. Math. Anal. 5 (1974), 19-29.

[9] F. W. J. OLVER, Uniform, exponentially improved, asymptotic expansions for the generalized exponential integral, SIAM J. Math. Anal. 22 (1991), 1460-1474.

[10] F. W. J. OLVER, Uniform, exponentially improved, asymptotic expansions for the Confluent Hypergeometric function and other integral transforms, SIAM J. Math. Anal. 22 (1991), 1475-1489.

[11] F. W. J. Olver, D. W. Lozier, R. F. Boisvert and C. W. Clark (eds.), NiST Handbook of Mathematical Functions, Cambridge University Press, New York, 2010.

[12] R. B. PARIS AND D. KAMINSKI, Asymptotics and Mellin-Barnes Integrals, Cambridge University Press, 2001. 